\title{
Active control of sound radiation from a simply supported beam: Influence of bending near-field waves
}

\author{
C. Guigou and C. R. Fuller \\ Vibration and Acoustic Laboratories, Mechanical Engineering Department, Virginia Polytechnic Institute \\ and State University, Blacksburg, Virginia 24061-0238
}

(Received 21 April 1992; revised 2 November 1992; accepted 4 December 1992)

\begin{abstract}
Active control of sound radiation from a baffled simply supported finite beam is analytically studied. The beam is subjected to a harmonic input force and the resulting acoustic field is minimized by applying a control point force. For a single frequency, the flexural response of the beam subject to the input and control forces is expressed in terms of flexural waves of both propagating and near-field types. The optimal control force complex amplitude is derived by minimizing the acoustic radiated pressure at one point located in the far field. The far-field radiated pressure, the displacement of the vibrating beam, and the one-dimensional wave-number spectrum of the beam velocity are extensively studied. In order to further understand control mechanisms, the radiated pressure due to the flexural propagating wave and the flexural near-field wave, respectively, is investigated at the minimization point before and after the control is involved. The analysis shows that, when the control is applied, the combination of the radiated pressure due to the two different types of waves (as their associated radiation is out-of-phase) at the minimization point causes the large pressure attenuation. These results demonstrate that structural near fields are important in terms of predicting performance in active control of structurally radiated sound.
\end{abstract}

PACS numbers: $43.40 . \mathrm{Vn}$

\section{INTRODUCTION}

Structural vibrations as well as their associated radiated sound fields have always been a problem in industry. If the sound radiation is unacceptable, different passive control approach are traditionally used to attenuate noise. However, passive techniques generally give poor control performances in the low-frequency region. In the last decade, active noise control has emerged as a practical possibility to reduce acoustic noise fields especially at low frequencies. This method usually employs acoustic control sources to attenuate the primary noise field. The active noise control approach and some of its applications have been described in Refs. 1 and 2. The technique has been successfully implemented for one-dimensional sound field. However, for a three-dimensional radiated sound field, there are some disadvantages with using acoustic sources as the control inputs amongst which are the number of sources required, size, and difficulties in implementation.

A new approach for the active control of structurally radiated sound fields has been investigated. Fuller ${ }^{3}$ demonstrated that reduction of the far-field acoustic radiation can also be obtained by directly modifying the response of the structure by applying structural inputs rather than by adding a distribution of acoustic sources in the surrounding sound field. The active control of sound radiation can be achieved by either point forces ${ }^{3}$ or piezoelectric elements ${ }^{4}$ directly applied on the vibrating structure surface, while information is obtained from radiation associated responses.
Most of all previous theoretical and experimental works on beams have been concerned with active control of bending motion and flexural power flow. ${ }^{5-10}$ The active control of sound radiation due to a clamped edge discontinuity on a semi-infinite beam has been investigated theoretically by Guigou and Fuller ${ }^{11}$ and experimentally by Guigou et al. ${ }^{12}$ Recently, the dynamic behavior of a controlled simply supported beam has been studied by Burdisso and Fuller. ${ }^{13,14}$ They demonstrated that the simply supported beam has new eigenproperties to the disturbance when the control is applied to minimize either the out-ofplane motion at one point on the beam or the radiated pressure level at one point in space.

In the present paper, active control of sound radiation from a finite simply supported beam is studied. The beam is subjected to a harmonic input force and the resulting acoustic field is minimized by applying a control point force (both forces approximating shakers). For a single frequency, the flexural response of the beam subject to the input and control forces is expressed in terms of flexural waves of both propagating and near-field types. The optimal control force complex amplitude is derived by minimizing the acoustic radiated pressure at one point located in the far field. On determining the optimal control force, it can be re-substituted in the constitutive equations for the system and the minimized fields can be evaluated. The main scope of this paper is to investigate how the control of the radiated pressure at the minimization point occurs. It should be noted that all previous analytical studies on ac- 


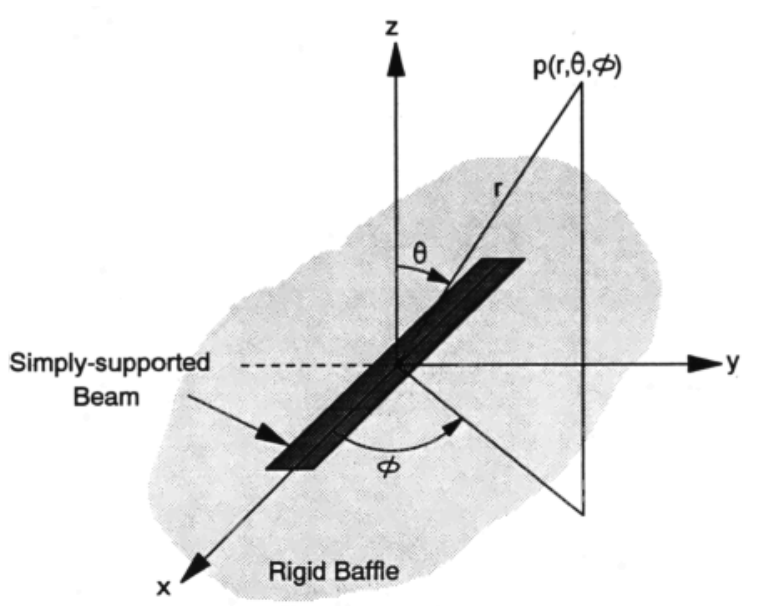

FIG. 1. Coordinate system.

tive structural acoustic control (ASAC), for example Ref. 3 , have ignored structural near fields. The main purpose of the present paper is to determine whether these structural near fields, present at discontinuities such as the control and input locations and the boundaries, are important in determining and predicting control performance. To this end, the radiated pressure due to the flexural propagating wave and the flexural near-field wave, respectively, is compared at the minimization point before and after the control is involved and the importance of the wave components is investigated.

\section{BEAM DISPLACEMENT}

The Cartesian coordinate system used in this analysis and the location of the simply supported beam in the coordinate system are shown in Fig. 1. The beam is taken to be located in an infinite baffle (in the $x, y$ plane) and the simply supported boundary conditions are applied at both ends, i.e., at $x= \pm l / 2$, where $l$ is the length of the beam. The time dependence for all the fields is assumed to be $e^{-i \omega t}$, where $\omega$ is the angular frequency.

The simply supported beam is first excited by an input shaker, modeled as a point force $\hat{F}_{n}$ located at $x=\alpha_{n}$. To achieve active control, a control point force $\hat{F}_{c}$ located at $x=\alpha_{c}$, approximating a control shaker, is then applied to the beam. This system is described in Fig. 2(a).

Consider an infinitely long beam. Here, $\hat{F}_{1}, \hat{M}_{1}$ and $\hat{F}_{2}$, $\hat{M}_{2}$ applied on the infinite beam in the manner shown in Fig. 2(b) with the correct complex values will create at $x=-l / 2$ and $x=l / 2$, respectively, the boundary conditions of a simply supported beam. Hence, the part of the infinitely long beam, which is located between $-l / 2 \leqslant x \leqslant l /$ 2 , will behave in every respect as if there were simply supported conditions at both end. Therefore, the response of the beam due to

(i) an input point force $\hat{F}_{n}$ located at $x=\alpha_{n}$ (the disturbance),

(ii) a point force $\hat{F}_{1}$ located at $x=-l / 2$ (due to the simply supported condition), (a)

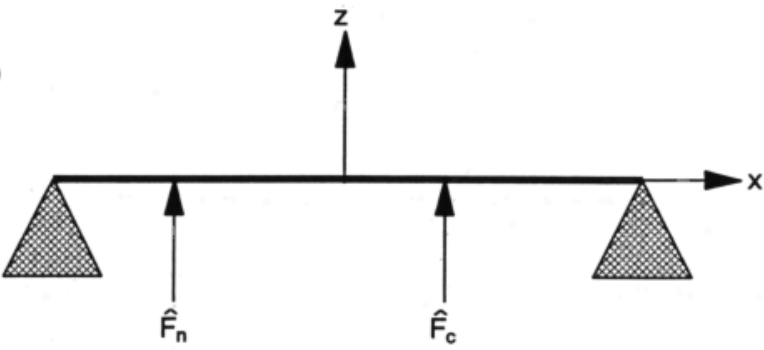

(b)

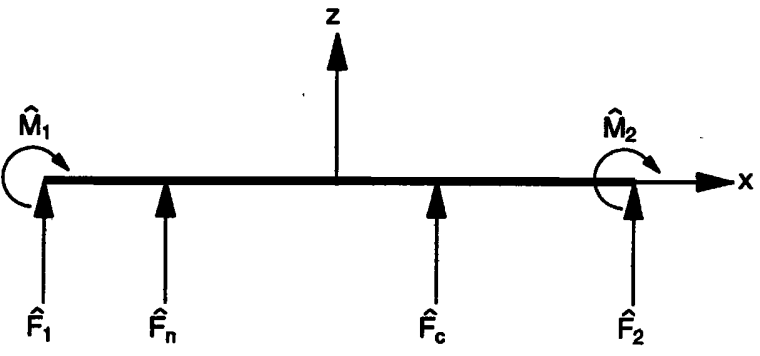

FIG. 2. (a) Schematic of the simply supported beam. (b) Forces and moments applied on the beam.

(iii) a line moment $\hat{M}_{1}$ located at $x=-l / 2$ (due to the simply supported condition),

(iv) a point force $\hat{F}_{2}$ located at $x=l / 2$ (due to the simply supported condition),

(v) a line moment $\hat{M}_{2}$ located at $x=l / 2$ (due to the simply supported condition),

(vi) and a control point force $\hat{F}_{c}$ located at $x=\alpha_{c}$, has to be derived.

The flexural displacements of an infinite thin beam due to a point force and a line moment have been fully derived by Guigou. ${ }^{11}$ It was found that the out-of-plane displacement due to a point force $\hat{F}$ located at any position $x=\alpha$ on the beam is

$$
w(x)=\left(i \hat{F} / 4 E I k_{J}^{3}\right)\left[e^{i k_{f}|x-\alpha|}+i e^{-k_{f}|x-\alpha|}\right]
$$

and the out-of-plane displacement due to a line moment located at any position $x=\alpha$ is

$$
w(x)=-\frac{\hat{M} \operatorname{sgn}(x-\alpha)}{E I k_{f}^{2}}\left[e^{i k_{f}|x-\alpha|}-e^{-k_{f}|x-\alpha|}\right],
$$

where the structural wave number is

$$
k_{f}=\sqrt[4]{\omega^{2} m / E I},
$$

$E I$ is the flexural stiffness ( $E$ is the modulus of elasticity, $I$ is the cross-sectional moment of inertia), $m$ is the density per unit length, both constant throughout the length of the beam, and the function $\operatorname{sgn}(x-\alpha)$ is defined by 


$$
\operatorname{sgn}(x-\alpha)= \begin{cases}+1, & \text { if } x \geqslant \alpha, \\ -1, & \text { otherwise. }\end{cases}
$$

It can be observed that a point force or a line moment creates two different flexural waves on each side of the application point: a propagating flexural wave (first term in the bracket) and a nonpropagating near-field flexural wave (second term in the bracket).

In this case, the beam is assumed to be slightly damped. Structural damping is included by using a complex modulus of elasticity defined as follows:

$$
\hat{E}=E(1-i \beta),
$$

where $\beta$ is the damping ratio or loss factor. The previous equations are also valid when $E$ is replaced by the complex modulus of elasticity. It can be noted that the structural bending wave number $k_{f}$ also becomes complex due to the damping.

Thus, using the superposition principle and Eqs. (1) and (2), the flexural displacement of the beam system as shown in Fig. 2(b) is given, for $-l / 2 \leqslant x \leqslant l / 2$, by

$$
\begin{aligned}
w(x)= & \frac{i \hat{F}_{n}}{4 \hat{E} I k_{f}^{3}}\left[e^{i k_{f}\left|x-\alpha_{n}\right|}+i e^{-k_{f}\left|x-\alpha_{n}\right|}\right]+\frac{i \hat{F}_{1}}{4 \hat{E} I k_{f}^{3}}\left[e^{i k_{f}(x+l / 2)}+i e^{-k_{f}(x+l / 2)}\right]-\frac{\hat{M}_{1}}{4 \hat{E} I k_{f}^{2}}\left[e^{i k_{f}(x+l / 2)}-e^{-k_{f}(x+l / 2)}\right] \\
& +\frac{i \hat{F}_{2}}{4 \hat{E} I k_{f}^{3}}\left[e^{i k_{f}(I / 2-x)}+i e^{-k_{f}(l / 2-x)}\right]+\frac{\hat{M}_{2}}{4 \hat{E} I k_{f}^{2}}\left[e^{i k_{f}(l / 2-x)}-e^{-k_{f}(l / 2-x)}\right]+\frac{i \hat{F}_{c}}{4 \hat{E} I k_{f}^{3}}\left[e^{i k_{f}\left|x-\alpha_{c}\right|}+i e^{-k_{f}\left|x-\alpha_{c}\right|}\right] .
\end{aligned}
$$

Note, in Eq. (6) positive and negative wave components due to the noise and control forces are derivable from the modulus of the spatial variation term.

The forces, $\hat{F}_{1}$ and $\hat{F}_{2}$, and the moments, $\hat{M}_{1}$ and $\hat{M}_{2}$, are found in such a way that the system satisfies the simply supported boundary conditions at $x=-l / 2$ and at $x=l / 2$, which are defined by

$$
\left.w\right|_{x=-l / 2}=\left.w\right|_{x=l / 2}=0
$$

and

$$
\left.\frac{\partial^{2} w}{\partial x^{2}}\right|_{x=-l / 2}=\left.\frac{\partial^{2} w}{\partial x^{2}}\right|_{x=l / 2}=0 .
$$

Then, the four unknowns magnitudes are given by a system of four linear equations, which can be written in matrix form as follows:

$$
\begin{gathered}
{\left[\begin{array}{cccc}
(1+i) & \left(e^{i k_{f} l}+i e^{-k_{f} l}\right) & 0 & -i k_{f}\left(e^{i k_{f} l}-e^{-k_{f} l}\right) \\
\left(e^{i k_{f} l}+i e^{-k_{f} l}\right) & (1+i) & i k_{f}\left(e^{i k_{f} l}-e^{-k_{f} l}\right) & 0 \\
(-1+i) & \left(-e^{i k_{f} l}+i e^{-k_{f} l}\right) & -2 i k_{f} & i k_{f}\left(e^{i k_{f} l}+e^{-k_{f} l}\right) \\
\left(-e^{i k_{f} l}+i e^{-k_{f} l}\right) & (-1+i) & -i k_{f}\left(e^{i k_{f} l}+e^{-k_{f} l}\right) & 2 i k_{f}
\end{array}\right]\left[\begin{array}{l}
\hat{F}_{1} \\
\hat{F}_{2} \\
\hat{M}_{1} \\
\hat{M}_{2}
\end{array}\right]} \\
=\left[\begin{array}{c}
-\left(e^{i k_{f}\left(l / 2+\alpha_{n}\right)}+i e^{-k_{f}\left(l / 2+\alpha_{n}\right)}\right) \\
-\left(e^{i k_{f}\left(l / 2-\alpha_{n}\right)}+i e^{-k_{f}\left(l / 2-\alpha_{n}\right)}\right) \\
-\left(-e^{i k_{f}\left(l / 2+\alpha_{n}\right)}+i e^{-k_{f}\left(l / 2+\alpha_{n}\right)}\right) \\
-\left(-e^{i k_{f}\left(l / 2-\alpha_{n}\right)}+i e^{-k_{f}\left(l / 2-\alpha_{n}\right)}\right)
\end{array}\right] \hat{F}_{n}+\left[\begin{array}{c}
-\left(e^{i k_{f}\left(l / 2+\alpha_{c}\right)}+i e^{-k_{f}\left(l / 2+\alpha_{c}\right)}\right) \\
-\left(e^{i k_{f}\left(l / 2-\alpha_{c}\right)}+i e^{-k_{f}\left(l / 2-\alpha_{c}\right)}\right) \\
-\left(-e^{i k_{f}\left(l / 2+\alpha_{c}\right)}+i e^{-k_{f}\left(l / 2+\alpha_{c}\right)}\right) \\
-\left(-e^{i k_{f}\left(l / 2-\alpha_{c}\right)}+i e^{-k_{f}\left(l / 2-\alpha_{c}\right)}\right)
\end{array}\right] \hat{F}_{c} .
\end{gathered}
$$

Therefore, the four unknowns $\hat{F}_{1}, \hat{F}_{2}, \hat{M}_{1}$, and $\hat{M}_{2}$ are determined as functions of the input point force $\hat{F}_{n}$ and the control point force $\hat{F}_{c}$. For simplification of the notation, the unknowns are given by

$$
\left[\begin{array}{c}
\hat{F}_{1} \\
\hat{F}_{2} \\
\hat{M}_{1} \\
\hat{M}_{2}
\end{array}\right]=\left[\begin{array}{c}
A_{n}(1) \\
A_{n}(2) \\
A_{n}(3) \\
A_{n}(4)
\end{array}\right] \hat{F}_{n}+\left[\begin{array}{c}
A_{c}(1) \\
A_{c}(2) \\
A_{c}(3) \\
A_{c}(4)
\end{array}\right] \hat{F}_{c},
$$


where the $\dot{A}_{n}(j)$ and $A_{c}(j)$ for $j=1,4$ can be easily deduced from Eq. (9).

Thus, the flexural displacement of the simply supported beam is given by the following expression, for $-l / 2 \leqslant x \leqslant l / 2$ :

$$
\begin{aligned}
w(x)= & \left(i \hat{F}_{n} / 4 \hat{E} I k_{f}^{3}\right)\left[e^{i k_{f}\left|x-\alpha_{n}\right|}+i e^{-k_{f}\left|x-\alpha_{n}\right|}+\left(A_{n}(1)+i k_{f} A_{n}(3)\right) e^{i k_{f}(x+l / 2)}+\left(i A_{n}(1)-i k_{f} A_{n}(3)\right) e^{-k_{f}(x+l / 2)}\right. \\
& \left.+\left(A_{n}(2)-i k_{f} A_{n}(4)\right) e^{i k_{f}(l / 2-x)}+\left(i A_{n}(2)+i k_{f} A_{n}(4)\right) e^{-k_{f}(l / 2-x)}\right]+\left(i \hat{F}_{c} / 4 \hat{E} I k_{f}^{3}\right)\left[e^{i k_{f}\left|x-\alpha_{c}\right|}+i e^{-k_{f}\left|x-\alpha_{c}\right|}\right. \\
& +\left(A_{c}(1)+i k_{f} A_{c}(3)\right) e^{i k_{f}(x+l / 2)}+\left(i A_{c}(1)-i k_{f} A_{c}(3)\right) e^{-k_{f}(x+l / 2)}+\left(A_{c}(2)-i k_{f} A_{c}(4)\right) e^{i k_{f}(l / 2-x)} \\
& \left.+\left(i A_{c}(2)+i k_{f} A_{c}(4)\right) e^{-k_{f}(l / 2-x)}\right] .
\end{aligned}
$$

It should be noted at this point that a simply supported boundary condition only transforms an incident (nearfield) propagating wave into a reflected propagating (nearfield) wave; traveling waves incident on a simple support do not generate a reflected near-field (unlike a clamped boundary condition, which couples an incident propagating wave with a reflected propagating wave and a reflected near-field wave). Thus, in this case, the input force (disturbance) and control force are mainly responsible for the presence of near-field flexural waves, except when located close to a boundary.

In terms of radiation, the simply supported beam system is considered as a 2-D structure and thus the displacement is constant along the width of the beam. Hence, the out-of-plane displacement can be rewritten as

$$
w(x, y)=w(x),
$$

for $|x| \leqslant l / 2$ and $|y| \leqslant l_{y} / 2$, where $l_{y}$ represents the width of the beam.

\section{FAR-FIELD RADIATED PRESSURE}

Using the spherical coordinate system as shown in Fig. 1, it was demonstrated in Ref. 11 that, for any general 2-D system with light fluid loading, the far-field radiated pressure can be closely approximated by the expression, derived using the method of stationary phase

$$
\begin{aligned}
p(r, \theta, \phi)= & -i \omega \rho_{0} \widetilde{v}\left(k_{0} \sin \theta \cos \phi, k_{0} \sin \theta \sin \phi\right) \\
& \times\left(e^{i k_{0} r} / 2 \pi r\right),
\end{aligned}
$$

where $\rho_{0}$ and $k_{0}$ are, respectively, the density and the wave number in the surrounding acoustic medium and $\widetilde{v}\left(k_{x}, k_{y}\right)$ represents the spatial Fourier transform of the velocity of the vibrating structure.

The far-field radiated pressure is a function of the double spatial Fourier transform of the out-of-plane beam velocity for $\sqrt{k_{x}^{2}+k_{y}^{2}} \leqslant k_{0}$, which is defined as the supersonic region. It can be noticed that a direction of radiation $(\hat{\theta}, \hat{\phi})$ in the far field is equivalent to a couple $\left(\hat{k}_{x}, \hat{k}_{y}\right)$ in the wave-number domain defined by

$$
\begin{aligned}
& \hat{k}_{x}=k_{0} \sin \hat{\theta} \cos \hat{\phi}, \\
& \hat{k}_{y}=k_{0} \sin \hat{\theta} \sin \hat{\phi},
\end{aligned}
$$

which are the points of stationary phase. It is now necessary to find the spectral response of the simply supported beam under study.

The double spatial Fourier transform is defined by

$$
\widetilde{v}\left(k_{x}, k_{y}\right)=\int_{-\infty}^{+\infty} \int_{-\infty}^{+\infty} v(x, y) e^{i k_{x} x} e^{i k_{y} y} d x d y,
$$

which is reduced to

$$
\widetilde{v}\left(k_{x}, k_{y}\right)=\int_{-l / 2}^{l / 2} \int_{-l_{y} / 2}^{l_{y} / 2} v(x, y) e^{i k_{x} x} e^{i k_{y} y} d y d x
$$

as the beam is located in a rigid infinite baffle, i.e., $v(x, y)=0$ for $|x|>l / 2$ and $|y|>l_{y} / 2$.

As the beam displacement is taken to be constant along the width of the beam [see Eq. (12)], the integrals with respect to $x$ and $y$ are separable, i.e.,

$$
\begin{aligned}
\tilde{v}\left(k_{x}, k_{y}\right) & =\int_{l_{y} / 2}^{l_{y} / 2} e^{i k_{y} y} d y \int_{-l / 2}^{l / 2} v(x) e^{i k_{x} x} d x \\
& =\tilde{v}\left(k_{y}\right) \tilde{v}\left(k_{x}\right),
\end{aligned}
$$

where $v(x)$ can be derived from Eq. (11).

The expression of $\tilde{v}\left(k_{y}\right)$ is easily obtained by integration,

$$
\widetilde{v}\left(k_{y}\right)=\frac{\sin \left(k_{y} l_{y} / 2\right)}{k_{y} / 2},
$$

and the expression of $\tilde{v}\left(k_{x}\right)$ is found to be 


$$
\begin{aligned}
& \tilde{v}\left(k_{x}\right)=\frac{i \omega \hat{F}_{n}}{4 \hat{E} I k_{f}^{3}}\left(\frac{e^{i k_{x} \alpha_{n}}-e^{i\left(k_{f}-k_{x}\right)(1 / 2)+i k_{f} \alpha_{n}}}{k_{f}-k_{x}}+\frac{e^{i k_{x} \alpha_{n}}-e^{i\left(k_{f}+k_{x}\right)(1 / 2)-i k_{f} \alpha_{n}}}{k_{f}+k_{x}}+\frac{e^{i k_{x} \alpha_{n}}-e^{-\left(k_{f}+i k_{x}\right)(1 / 2)-k_{f} \alpha_{n}}}{k_{f}+i k_{x}}\right. \\
& +\frac{e^{i k_{x} \alpha_{n}}-e^{-\left(k_{f}-i k_{x}\right)(l / 2)+k_{f} \alpha_{n}}}{k_{f}-i k_{x}}+\left(A_{n}(1)+i k_{f} A_{n}(3)\right) e^{-i k_{x}(l / 2)} \frac{1-e^{i\left(k_{f}+k_{x}\right) l}}{k_{f}+k_{x}} \\
& +\left(A_{n}(1)-k_{f} A_{n}(3)\right) e^{-i k_{x}(l / 2)} \frac{1-e^{-\left(k_{f}-i k_{x}\right) l}}{k_{f}-i k_{x}}+\left(A_{n}(2)-i k_{f} A_{n}(4)\right) e^{i k_{x}(l / 2)} \frac{1-e^{i\left(k_{f}-k_{x}\right) l}}{k_{f}-k_{x}} \\
& \left.+\left(A_{n}(2)+k_{f} A_{n}(4)\right) e^{i k_{x}(1 / 2)} \frac{1-e^{-\left(k_{f}+i k_{x}\right) l}}{k_{f}+i k_{x}}\right)+\frac{i \omega \hat{F}_{c}}{4 \hat{E} I k_{f}^{3}}\left(\frac{e^{i k_{x} a_{c}}-e^{i\left(k_{f}-k_{x}\right)(1 / 2)+i k_{f} \alpha_{c}}}{k_{f}-k_{x}}\right. \\
& +\frac{e^{i k_{x} \alpha_{c}}-e^{i\left(k_{f}+k_{x}\right)(1 / 2)-i k_{f} \alpha_{c}}}{k_{f}+k_{x}}+\frac{e^{i k_{x} \alpha_{c}}-e^{-\left(k_{f}+i k_{x}\right)(1 / 2)-k_{f} \alpha_{c}}}{k_{f}+i k_{x}}+\frac{e^{i k_{x} \alpha_{c}}-e^{-\left(k_{f}-i k_{x}\right)(l / 2)+k_{f} \alpha_{c}}}{k_{f}-i k_{x}} \\
& +\left(A_{c}(1)+i k_{f} A_{c}(3)\right) e^{-i k_{x}(l / 2)} \frac{1-e^{i\left(k_{f}+k_{x}\right) l}}{k_{f}+k_{x}}+\left(A_{c}(1)-k_{f} A_{c}(3)\right) e^{-i k_{x}(l / 2)} \frac{1-e^{-\left(k_{f}-i k_{x}\right) l}}{k_{f}-i k_{x}} \\
& \left.+\left(A_{c}(2)-i k_{f} A_{c}(4)\right) e^{i k_{x}(1 / 2)} \frac{1-e^{i\left(k_{f}-k_{x}\right) l}}{k_{f}-k_{x}}+\left(A_{c}(2)+k_{f} A_{c}(4)\right) e^{i k_{x}(l / 2)} \frac{1-e^{-\left(k_{f}+i k_{x}\right) l}}{k_{f}+i k_{x}}\right) .
\end{aligned}
$$

The method of separating the double spatial Fourier integral into two single integrals, implies that $\widetilde{v}\left(k_{y}\right)$ is independent of the input force and the control force. Thus, only the one-dimensional wave-number spectrum $\widetilde{v}\left(k_{x}\right)$ will be studied before and after the control is applied.

\section{OPTIMAL CONTROL}

In the following control strategy, the sound pressure level is to be minimized at one specified point in the far field. The location of the point in space, where the sound pressure level has to be optimized, is given by $\left(r_{e}, \theta_{e}, \phi_{e}\right)$ in the spherical coordinate system. The control point force has to drive the beam such that the sound pressure level is minimum at this location. Thus, the optimum complex amplitude of the control point force $\hat{F}_{c}$ is obtained by minimizing the square value of the pressure modulus at the point $\left(r_{e}, \theta_{e}, \phi_{e}\right)$ in the far field.

The cost function, defined as the square of the pressure modulus is

$$
\Lambda=p\left(r_{e}, \theta_{e}, \phi_{e}\right) p^{*}\left(r_{e}, \theta_{e}, \phi_{e}\right),
$$

where "*" denotes the complex conjugate.

To simplify the notation, the far-field radiated pressure at $\left(r_{e}, \theta_{e}, \phi_{e}\right)$ can be rewritten as

$$
p\left(r_{e}, \theta_{e}, \phi_{e}\right)=N\left(r_{e}, \theta_{e}, \phi_{e}\right) \hat{F}_{n}+C\left(r_{e}, \theta_{e}, \phi_{e}\right) \hat{F}_{c},
$$

where the values of $N\left(r_{e}, \theta_{e}, \phi_{e}\right)$ and $C\left(r_{e}, \theta_{e}, \phi_{e}\right)$ can be deduced from Eqs. (13), (18), and (19).

Therefore, the cost function $\Lambda$ to be minimized is rewritten as

$$
\begin{aligned}
\Lambda= & N\left(r_{e}, \theta_{e}, \phi_{e}\right) N^{*}\left(r_{e}, \theta_{e}, \phi_{e}\right) \hat{F}_{n} \hat{F}_{n}^{*} \\
& +N\left(r_{e}, \theta_{e}, \phi_{e}\right) C^{*}\left(r_{e}, \theta_{e}, \phi_{e}\right) \hat{F}_{n} \hat{F}_{c}^{*} \\
& +N^{*}\left(r_{e}, \theta_{e}, \phi_{e}\right) C\left(r_{e}, \theta_{e}, \phi_{e}\right) \hat{F}_{n}^{*} \hat{F}_{c} \\
& +C\left(r_{e}, \theta_{e}, \phi_{e}\right) C^{*}\left(r_{e}, \theta_{e}, \phi_{e}\right) \hat{F}_{c} \hat{F}_{c}^{*} .
\end{aligned}
$$

The cost function is a real quadratic function of the control force. For a given location of the control force, this quadratic cost function has a unique minimum value associated with an optimal control force magnitude. Taking the derivatives of the cost function $\Lambda$ with respect to the real and imaginary part of the control force leads to the optimal solution for the control force complex amplitude

$$
\hat{F}_{c}=-\frac{N\left(r_{e}, \theta_{e}, \phi_{e}\right)}{C\left(r_{e}, \theta_{e}, \phi_{e}\right)} \hat{F}_{n} .
$$

It should be noticed that minimizing the radiated pressure in the far field at $\left(r_{e}, \theta_{e}, \phi_{e}\right)$ is equivalent to reducing the wave-number spectrum at $\left(k_{x e}, k_{y e}\right)$, where $k_{x e}$ and $k_{y e}$ are given by Eq. (14) as a function of $\theta_{e}$ and $\phi_{e}$. Wave-number domain control approaches based on this concept have been demonstrated in Ref. 15. Therefore, the control force is independent of the radius $r_{e}$, if $r_{e}$ is large enough to place the minimization point in the far field. Thus, this type of control is referred as a directional control, as the radiation is minimized in the direction $\left(\theta_{e}, \phi_{e}\right)$ in the far field.

\section{INDIVIDUAL FLEXURAL WAVE CONTRIBUTION}

Since the out-of-plane displacement of the beam is given in terms of both propagating and nonpropagating near-field waves, it is possible to determine their relative contribution to the radiated far-field pressure. 
TABLE I. Beam characteristics.

\begin{tabular}{lc}
\hline \hline Width & $4.0 \mathrm{~cm}$ \\
Length & $38.0 \mathrm{~cm}$ \\
Flexural stiffness & $5.33 \mathrm{~N} \mathrm{~m}$ \\
Density & $0.6267 \mathrm{~kg} / \mathrm{m}$ \\
Damping ratio & $0.1 \%$ \\
\hline
\end{tabular}

Equation (11), giving the out-of-plane displacement of the simply supported beam, can be rewritten in the form

$$
w(x)=w_{\mathrm{pr}}(x)+w_{\mathrm{nf}}(x),
$$

where $w_{\mathrm{pr}}$ and $w_{\mathrm{nf}}$ represent, respectively, the propagating (terms in $e^{ \pm i k_{f} x}$ ) and nonpropagating near-field (terms in $e^{ \pm k_{f} x}$ ) components of the beam flexural displacement. Upon taking the spatial Fourier transform of each displacement component, the far-field radiated pressure can be then expressed as

$$
p(r, \theta, \phi)=p_{\mathrm{pr}}(r, \theta, \phi)+p_{\mathrm{nf}}(r, \theta, \phi),
$$

where $p_{\mathrm{pr}}$ and $p_{\mathrm{nf}}$ are, respectively, the pressure radiated by the propagating and near-field component of the beam displacement. This decomposition of the pressure into two components respectively due to the propagating waves and to the near-field waves is expected to show the mechanisms by which the control is achieved at the minimization point, as well as enabling a study of the relative contributions of the different wave types.

\section{RESULTS}

The beam characteristics are presented in Table I. The input point force is taken to have a magnitude of $1 N$ and is located at $x=\alpha_{n}=-0.4 l$. The control force position is fixed at $x=\alpha_{c}=l / 8$. Three different frequencies of excitation are studied: $31.7 \mathrm{~Hz}$, corresponding to the first flexural mode of the system; $126 \mathrm{~Hz}$, corresponding to the second flexural mode of the system; and $600 \mathrm{~Hz}$, corresponding to an off-resonance frequency of the system. The radiated pressure is computed at a radius $r=7.62 \mathrm{~m}$ from the center of the beam, which is well into the far field. The pressure field is minimized at the location $\left(r_{e}=7.62 \mathrm{~m}, \theta_{e}=0, \phi_{e}\right.$ $=0$ ) in the spherical coordinate system, which is at a distance of $7.62 \mathrm{~m}$ above the center of the beam in the $x, z$ plane. By minimizing the radiated pressure in the far field at this location, the one-dimensional wave-number spectrum is minimized at $k_{x e}=0$, as explained previously.

\section{A. On-resonance excitation $31.7 \mathrm{~Hz}$, first mode}

Figure 3 shows the far-field pressure radiated in the $x, z$ plane by the simply supported beam for an excitation frequency of $31.7 \mathrm{~Hz}$, corresponding to the first mode. First, it can be noticed that, as expected for this frequency, the radiation from the beam, when no control is involved, is similar to a monopole source corresponding to the structural motion of the first mode. For this particular case, the radiated pressure due to the propagating flexural waves and the near-field flexural waves, respectively, is shown in Fig. 4(a) and is monopolelike for both of them. It can be seen that the radiation is mostly due to the propagating

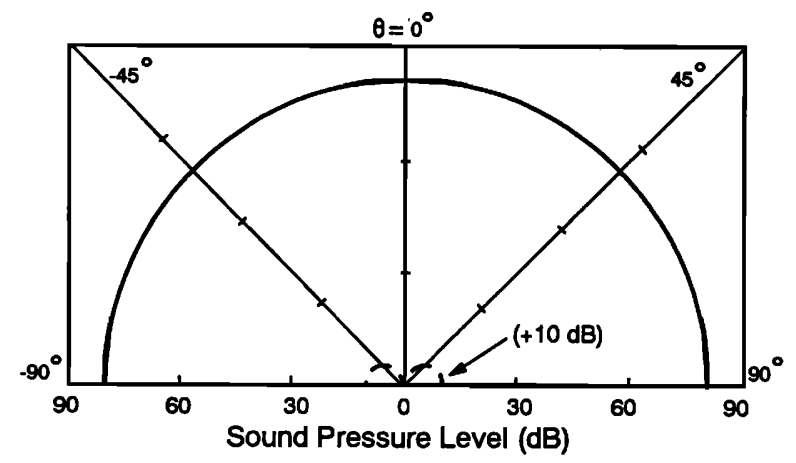

FIG. 3. Far-field pressure radiated in $x, z$ plane at $31.7 \mathrm{~Hz}$ - $\longrightarrow$, without control; - - with control.

waves and that the two different types of waves radiate in phase such that their associated radiated fields add coherently (as the total sound pressure level is larger than either each). When the control force is applied to the beam in order to attenuate the pressure at the minimization point, Fig. 3 shows that the pressure field is highly attenuated (more than $80-\mathrm{dB}$ reduction) and dipole like ( $10 \mathrm{~dB}$ were added to the actual pressure levels in order for the pressure field under control to be observable). In Fig. 4(b), it can be observed that the radiated pressure due to the propagating flexural wave has been attenuated by about $65 \mathrm{~dB}$, and that the radiated pressure due to the near-field waves has been reduced by $15 \mathrm{~dB}$. When the control is invoked, the sound pressure levels associated to these two types of waves are almost equal (about $15 \mathrm{~dB}$ in all directions).

(a)

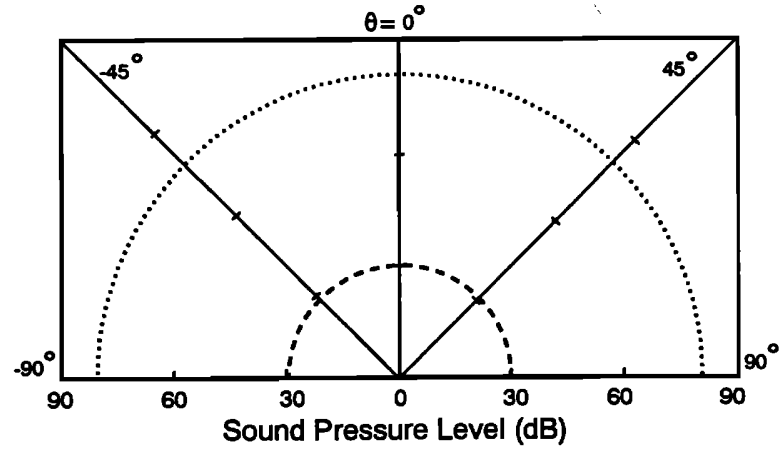

(b)

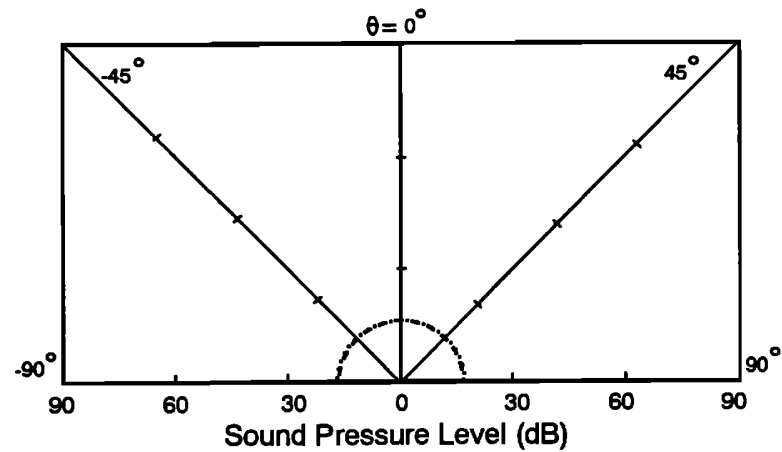

FIG. 4. Components of the far-field pressure radiated in $x, z$ plane at 31.7 Hz: (a) without control; (b) with control. - - -, due to near-field flexural waves; ----, due to propagating flexural waves. 


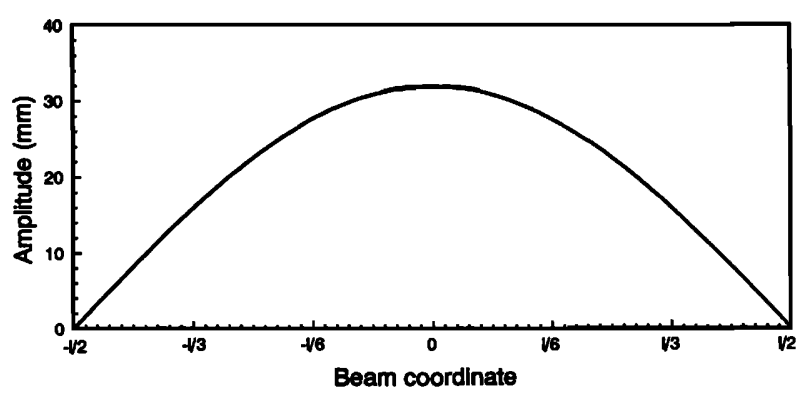

FIG. 5. Out-of-plane displacement at $31.7 \mathrm{~Hz}$ : —_, without control; - - with control.

However, as the total radiated pressure is largely attenuated, it can be deduced that these two different flexural waves now radiate out-of-phase, such that their associated radiated field cancel each other.

Figure 5 shows the out-of-plane displacement without and with control. (The large displacements are due to the slightly damped system being driven very close to resonance.) Before the control force is applied, the displacement corresponds to that expected, i.e., a mode shape corresponding to the first mode of the simply supported beam. When control is applied, the displacement of the beam is reduced to a very low level of vibration (hardly observable on the figure). Therefore, in this case, the attenuation of the radiated pressure is associated with a direct attenuation of the vibratory motion of the beam.

The one-dimensional wave-number spectrum $\left[\widetilde{v}\left(k_{x}\right)\right]$ is shown on Fig. 6. In the case without control, the spectrum corresponds exactly to the one expected for a fundamental mode, with a maximum of amplitude at $k_{x}=0$. When the control is involved, the wave-number spectrum amplitude is reduced to an amplitude close to zero for all the wave numbers. The extremely small amplitudes in the supersonic region (region defined as the radiating wavenumber components) correspond to the fact that the pressure is nearly completely attenuated (as noticed on Fig. 3 ).

\section{B. On-resonance excitation $126 \mathrm{~Hz}$, second mode}

For the second case, the beam is excited with a frequency of $126 \mathrm{~Hz}$ near the second mode of vibration. Figure 7 shows the far-field radiated pressure without and

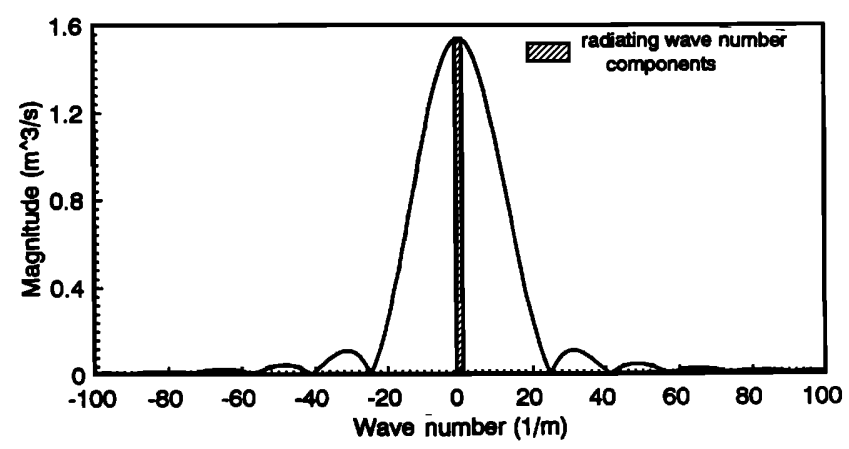

FIG. 6. Wave-number spectrum at $31.7 \mathrm{~Hz}$ : - , without control; - - with control.

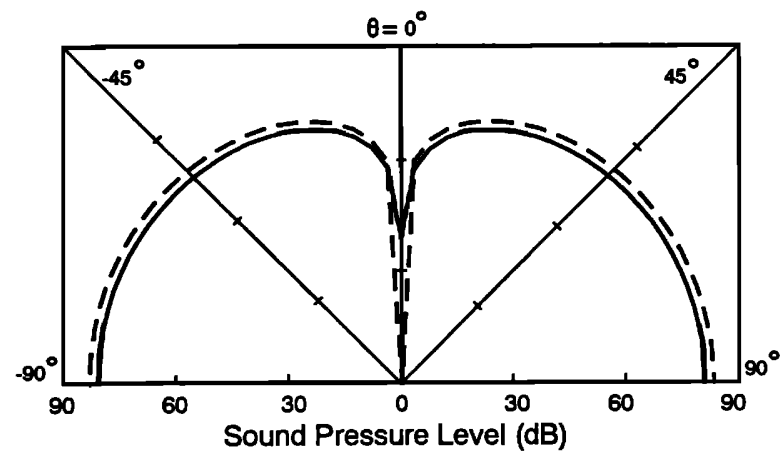

FIG. 7. Far-field pressure radiated in $x, z$ plane at $126 \mathrm{~Hz}$ : —, without control; - - with control.

with control. For the uncontrolled case, the radiation pattern is similar to that of a dipole radiator, as it is expected for the second mode of vibration. However, the sound pressure level is not zero at $\theta=0$, as the pressure radiated by the second mode should be. This is probably due to the fact that the vibratory motion has small components from other modes and so is not perfectly symmetric with respect to the center of the beam due to the nonsymmetric position of the disturbance force. Figure 8(a) shows that the propagating flexural wave is mostly responsible for the sound radiation in all direction except for $\theta$ close to zero. The near-field flexural waves still radiate as a monopole radiator even if the overall motion is dominantly dipole like. At $\theta=0$, these two types of waves radiate in phase. When the control is invoked, the sound pressure level is attenuated at

(a)

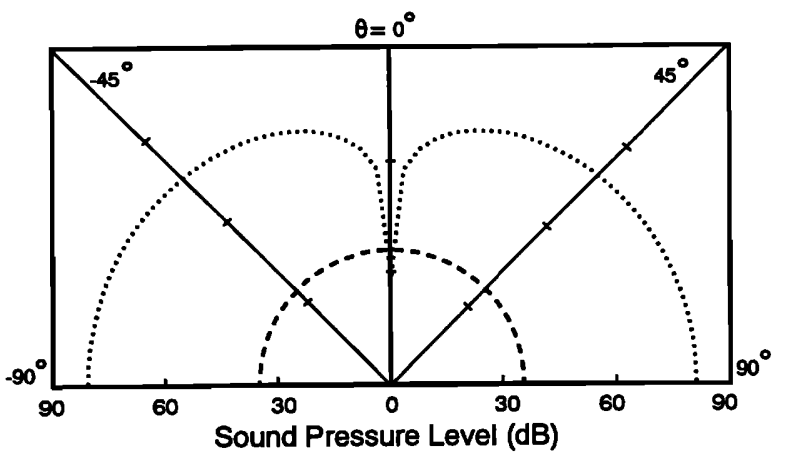

(b)

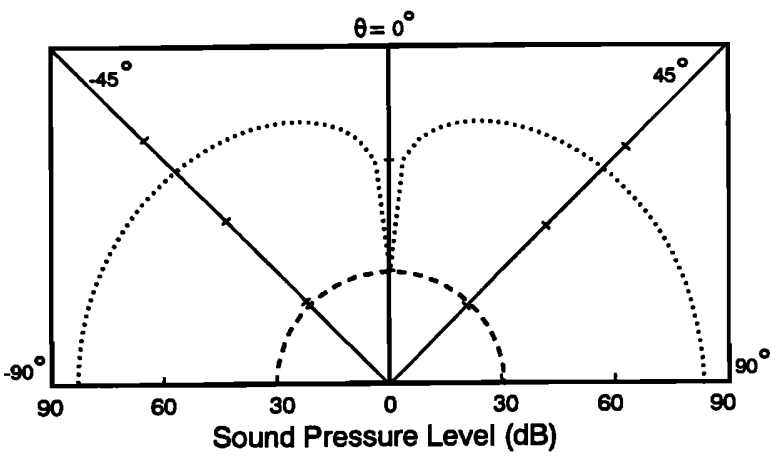

FIG. 8. Components of the far-field pressure radiated in $x, z$ plane at 126 Hz: (a) without control; (b) with control - - -, due to near-field flexural waves; -.-., due to propagating flexural waves. 


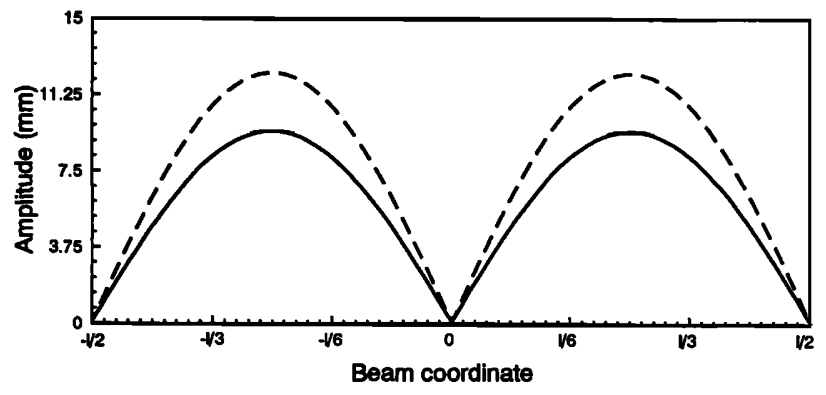

FIG. 9. Out-of-plane displacement at $126 \mathrm{~Hz}$ : — , without control; - - with control.

the minimization point and for $\theta$ very close to zero, elsewhere it is slightly increased (about $2.5 \mathrm{~dB}$ ). Figure 8(b) shows that the pressure field due to the near-field waves has been decreased by $6 \mathrm{~dB}$; on the other hand, the pressure field due to the propagating waves has been increased by about $2 \mathrm{~dB}$. However, at $\theta=0$, the acoustic pressure radiation due to the propagating waves and the one due to the near-field waves are equal and out-of-phase, such that the total radiated pressure at $\theta=0$ is canceled. This result implies that the attenuation at the error point is due to the interaction of the propagating and near-field waves; a result that stresses the importance of near-field components.

The out-of-plane displacement can be observed on Fig. 9. It corresponds, as expected, to the second mode of vibration, with a node at the center of the beam and a maximum displacement at $x= \pm l / 4$. When the control is applied, the mode shape is unchanged but the vibration level is increased. Thus, in this case, the attenuation of the pressure level at the minimization point in the far field leads to an increase of the vibratory motion. Note that near-field terms in the structural response are unobservable. However, being monopolelike, they are more efficient radiators than the propagating component, which increases the nearfield structural motion importance in the radiated acoustic field.

The one-dimensional wave-number spectrum for the uncontrolled and controlled cases can be observed on Fig. 10. The shaded region denotes the supersonic region. As expected for the uncontrolled case, the wave-number spectrum peaks for $k_{x} \simeq \pm\left|k_{f}\right|$, where $\left|k_{f}\right|$ is the modulus of the complex structural wave number. This maximum can

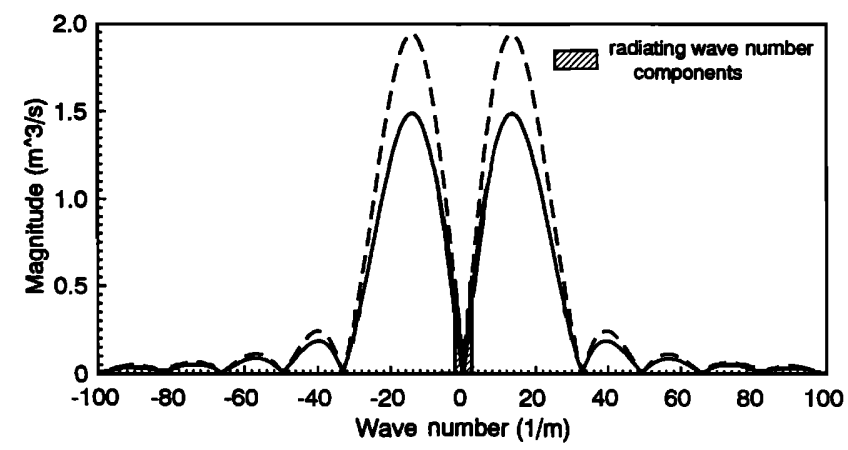

FIG. 10. Wave-number spectrum at $126 \mathrm{~Hz}$ : - , without control; - - with control.

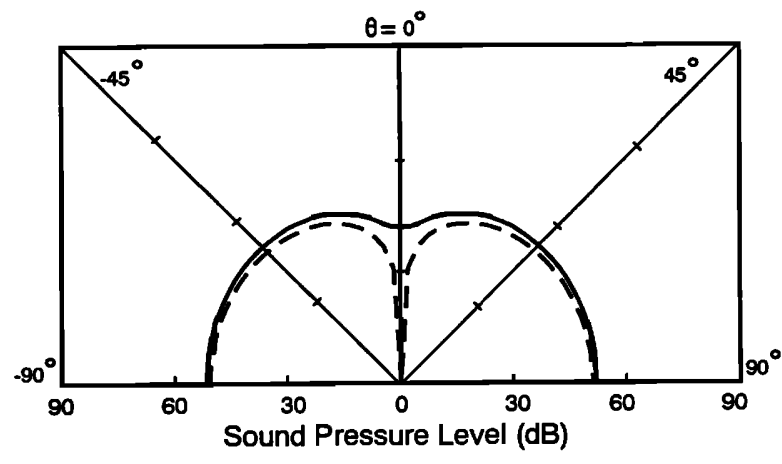

FIG. 11. Far-field pressure radiated in $x, z$ plane at $600 \mathrm{~Hz}$ : without control; $-\ldots$, with control.

also be related to $k_{x} \simeq \pm 2 \pi / l$, corresponding to the second flexural vibrational mode of the simply supported beam determined by modal analysis. When the control is applied, the amplitude of the wave-number spectrum is largely reduced at $k_{x}=0$ and is slightly increased for all other wave numbers. This increase of magnitude in the supersonic region and the large decrease at $k_{x}=0$ correspond to the observation made in Fig. 7. The spectrum still presents the same peaks, i.e., the mode shape of the beam is not changed by the control (as noticed previously in Fig. 9).

\section{Off-resonance excitation $600 \mathrm{~Hz}$}

The last case studied corresponds to an off-resonance excitation of the simply supported beam at $600 \mathrm{~Hz}$. Figure 11 shows the far-field radiated pressure without and with control. The radiation pattern without control is almost like a dipole radiation pattern with only a slight node around $\theta=0$, implying contributions from several modes. In Fig. 12(a), it can be noticed that the near-field flexural waves still radiate as a monopole radiator, and are radiating $12.5 \mathrm{~dB}$ more in level than the propagating waves at $\theta=0$. The two wave components radiate in phase and thus add coherently. When the control is applied, the far-field radiation from the beam is reduced to a dipole radiation structural motion, with no radiation in the direction $\theta=0$. The sound pressure levels are also well reduced for the directions close to $\theta=0$ and slightly attenuated elsewhere. It can be noted in Fig. 12(b) that at $\theta=0$, the radiation from the near-field waves has been reduced by $7.3 \mathrm{~dB}$ and the one from the propagating waves has been increased by $5 \mathrm{~dB}$. The attenuation of the total sound pressure level at $\theta=0$ again occurs because the two components radiate equally and out-of-phase.

Figure 13 shows the out-of-plane displacement, which is close to that corresponding to the fourth mode (507 $\mathrm{Hz}$ ). Three node and four antinodes can be observed; however, the displacement distribution is not symmetric with respect to the center of the beam. When the control is invoked, the mode shape is changed as the location of two nodes are moved, and the maximums of amplitude are attenuated. It can also be noticed that the displacement amplitude is attenuated between $x=l / 2$ and the location of the input force. In this case, the minimization process leads 
(a)

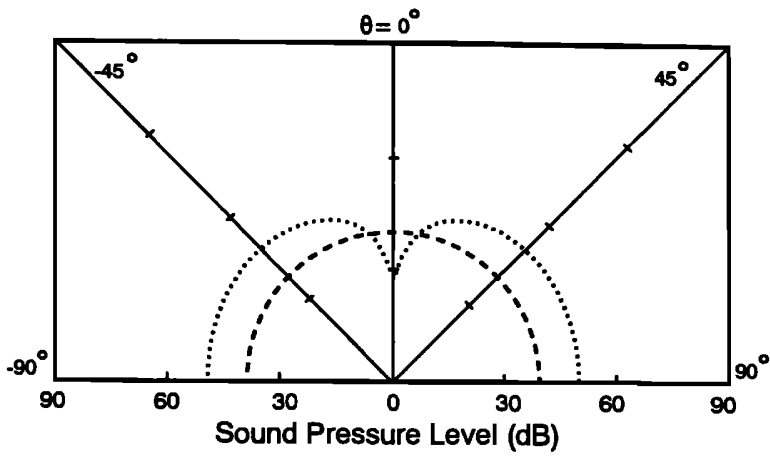

(b)

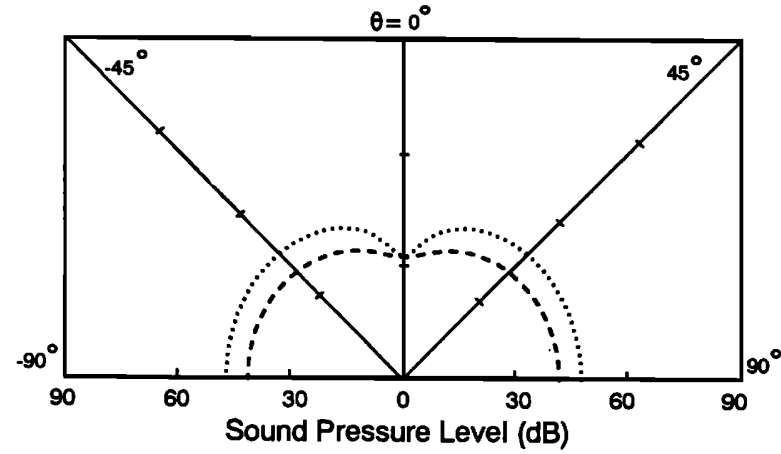

FIG. 12. Components of the far-field pressure radiated in $x, z$ plane at 600 $\mathrm{Hz}$; (a) without control; (b) with control. - - -, due to near-field flexural waves; ----, due to propagating flexural waves.

to a small decrease of radiation for directions not close to $\theta=0$ and to a change of mode shape and a decrease in amplitude of the out-of-plane displacement.

Figure 14 presents the one-dimensional wave-number spectrum with and without control. For the uncontrolled case, two main peaks can be observed close to $k_{x} \simeq \pm\left|k_{f}\right|$ and two other ones closer to $k_{x}=0$ located at $k_{x}= \pm\left|k_{f}\right| /$ 4 . The two main peaks, also corresponding to $k_{x} \simeq 4 \pi / l$ for modal analysis, shows that the fourth flexural vibrational mode is mostly responsible for the response of the beam at this frequency. When the control is invoked, the spectrum amplitude is reduced in all the supersonic region with a large attenuation at $k_{x}=0$. The general shape of the spectrum is changed (the values of $k_{x}$ for which the peaks occur are slightly increased), which is related to the change in the mode shape of the vibrating beam noticed previously.

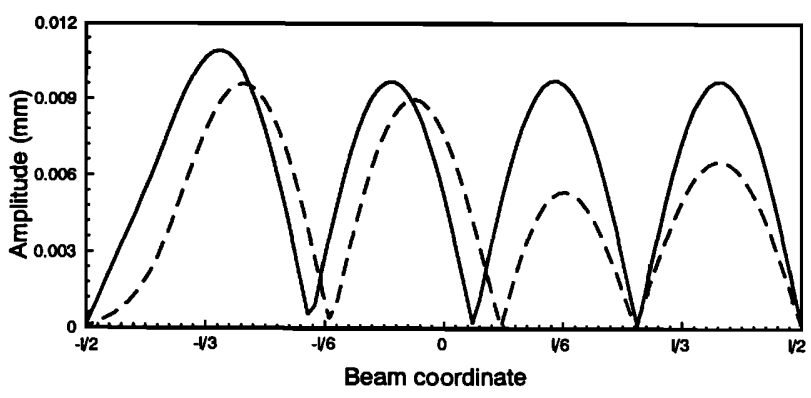

FIG. 13. Out-of-plane displacement at $600 \mathrm{~Hz}: \longrightarrow$, without control; - - with control.

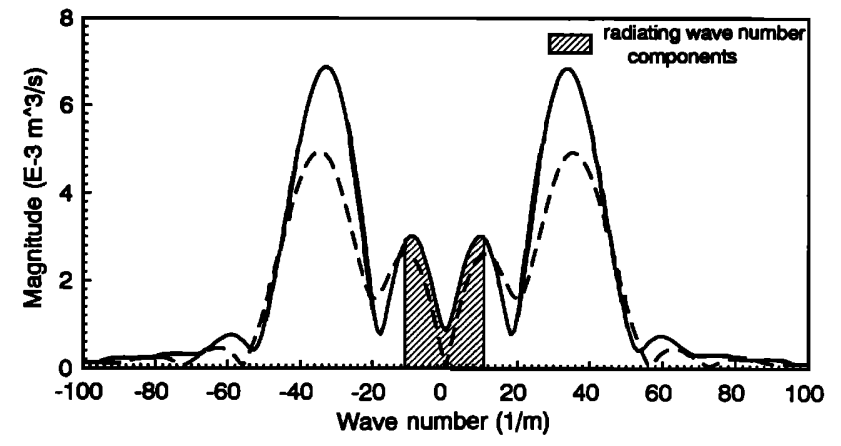

FIG. 14. Wave-number spectrum at $600 \mathrm{~Hz}$ : - , without control; - - with control.

\section{Control comparison with and without near-field waves}

It is interesting to investigate the control performance by calculating the optimal forces using only traveling waves and then calculate the minimized field using the expressions that include both near-field and traveling waves. Such calculations should give some insight on the consequences of neglecting the near field when predicting the performance of realistic ASAC system. Table II presents the amplitude and phase of the control forces in order to control, respectively, the total pressure field and the pressure field only due to the propagating waves in the direction $\theta=0$. First, it should be noticed that the control force is for all cases out-of-phase (phase close to $180^{\circ}$ ) with the input or disturbance force.

For the first mode of the simply supported beam, the control of either the total pressure or the pressure due to the propagating waves leads to almost the same optimal amplitude value for the control force. This was expected, as it was noticed in Fig. 4(a) that the propagating flexural waves are mainly responsible for the sound radiation at this frequency. When the control minimizes the pressure due to only propagating waves at $\theta=0$, the pressure due to the near-field waves is still reduced by half [in the same way observed in Fig. 4(b)] in all directions. However, the pressure due to the propagating waves becomes negligible as highly reduced in all directions. Therefore, the total pressure field under this control situation is approximately equal to the pressure field due to the near-field waves under control, which is about $16.5 \mathrm{~dB}$ in all directions.

For the second mode and off-resonance cases, Table II shows that the optimal control forces calculated with the

TABLE II. Optimal control force amplitude and phase.

\begin{tabular}{lccc}
\hline \hline Condition & $\begin{array}{c}\text { Frequency } \\
(\mathrm{Hz})\end{array}$ & $\begin{array}{c}\text { Force to control } \\
\text { pressureincl. both } \\
\text { wave types }\end{array}$ & $\begin{array}{c}\text { Force to control } \\
\text { pressure incl. only } \\
\text { propagating waves }\end{array}$ \\
\hline 1st mode & 31.7 & $0.334435 \mathrm{~N}$, & $0.334232 \mathrm{~N}$, \\
& & $\angle 179.99^{\circ}$ & $\angle 179.99^{\circ}$ \\
2nd mode & 126.0 & $0.252983 \mathrm{~N}$, & $0.112613 \mathrm{~N}$, \\
& & $\angle 179.98^{\circ}$ & $\angle 179.89^{\circ}$ \\
Off-resonance & 600.0 & $0.427298 \mathrm{~N}$, & $0.152152 \mathrm{~N}$, \\
& & $\angle 179.86^{\circ}$ & $\angle 179.30^{\circ}$ \\
\hline \hline
\end{tabular}




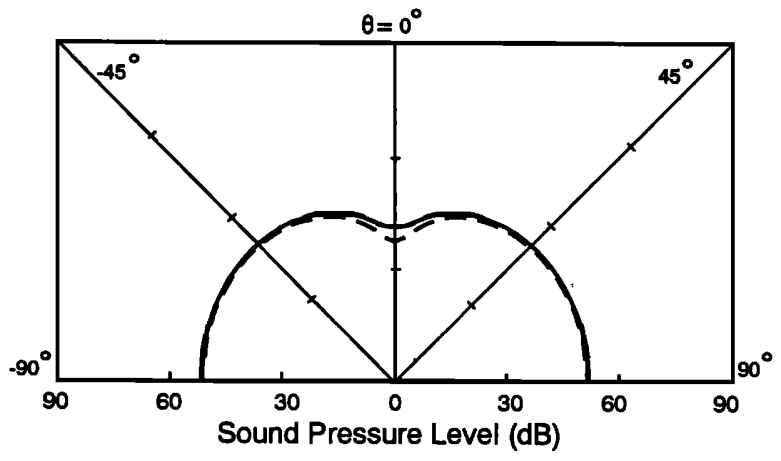

FIG. 15. Far-field pressure radiated in $x, z$ plane at $600 \mathrm{~Hz}$ : without control; $-\longrightarrow$, with control on propagating waves only.

near-field waves neglected are significantly different than when both wave types are included (corresponding to the realistic situation). Figure 15 presents the controlled radiation directivity for the frequency of $600 \mathrm{~Hz}$, corresponding to the optimal gains derived from only propagating wave information. When compared with Fig. 11, it is apparent that the main effect of ignoring the near-field waves on calculating the optimal gain is to markedly reduce the attenuation obtained at the error microphone. At other locations, the residual sound levels are increased by approximately $3 \mathrm{~dB}$. Calculations reveal that the residual value at the error microphone in Fig. 15 is equal to the near-field contribution at this point. This observation agrees with the usual observability requirements in control system performances (i.e., to be controlled, a variable has to be first observed).

\section{CONCLUSIONS}

The active control of sound radiation from a simply supported beam has been analytically studied. For excitation on- and off-resonance, a control point force approximating a shaker has been shown to be effective to actively modify the vibrational response of the system in order to obtain directional sound control, i.e., the minimization of the acoustic radiated pressure at one point located in the far field. The large decrease of the radiated pressure at the minimization point has been shown to be caused by the destructive combination of the pressure radiated, respectively, by the propagating flexural waves and the nonpropagating near-field flexural waves (equal and out-of-phase). This phenomenon proves that, even if the near-field flexural waves can be neglected in terms of vibration, they play an important role in terms of radiation and control. This characteristic is due to the high radiation efficiency associated with the monopole source term of the structural near fields. The attenuation of the radiated acoustic pressure at the minimization point in the far field is related to a decrease of the supersonic wave-number component, corresponding to the direction of minimization, in the vibrational response of the system. This attenuation is not necessarily associated with a decrease of the amplitude in the global vibrational response of the system. These results, in general, demonstrate that structural near fields play an important role in active structural acoustic control and should be considered in the design of ASAC systems.

\section{ACKNOWLEDGMENTS}

The authors wish to acknowledge the support of the sponsor of this work, the Office of Naval Research under Grant No. ONR-N00014-92-J-1170.

${ }^{1}$ G. E. Warnaka, "Active attenuation of noise- The state of the art," Noise Control Eng. 18(3), 110 (1982).

${ }^{2}$ J. E. Ffowcs-Williams, “Anti-sound," Proc. R. Soc. London Ser. A 395, 63-88 (1984).

${ }^{3}$ C. R. Fuller, "Active control of sound transmission/radiation from elastic plates by vibrational inputs: I. Analysis," J. Sound Vib. 136(1), 1-15 (1990).

${ }^{4}$ R. L. Clark, and C. R. Fuller, "Experiments on active control of structurally radiated sound using multiple piezoceramic actuators," J. Acoust. Soc. Am. 91, 3313-3320 (1992).

${ }^{5}$ W. Redman-While, P. A. Nelson, and A. R. D. Curtis, "Experiments on active control of flexural wave power," J. Sound Vib. 112(1), 181187.

${ }^{6}$ A. H. Von Flotow and B. Shafer, "Wave absorbing controllers for flexible beam", J. Guidance, Control Dyn. 9(6), 673-680 (1986).

${ }^{7}$ S. J. Elliot, I. M. Stothers, and L. Billet, "Adaptive feedforward control of flexural waves propagating in a beam," Proc. Inst. Acoust. 12(1), 613-622 (1990).

${ }^{8} \mathrm{~J}$. Sheuren, "Active attenuation of bending waves in beams," Proc. Inst. Acoust. 12(1), 623-629 (1990).

${ }^{9}$ C. R. Fuller, G. P. Gibbs, and R. J. Silcox, "Active control of flexural and extensional waves in beams," J. Intell. Mater. Syst. Struct. 1(2), 235-247 (1990).

${ }^{10}$ L. O. Gonidou, "Active Control of Flexural Power Flow in Elastic Thin Beams," MS thesis, Department of Mechanical Engineering, Virginia Polytechnic Institute and State University (1989).

"C. Guigou and C. R. Fuller, "Active control of sound radiation from a semi-infinite beam with a clamped edge," J. Sound Vib. (to be published).

${ }^{12}$ C. Guigou, C. R. Fuller, and K. D. Frampton, "Experiments on active control of sound radiation from a clamped edge on a semi-infinite beam," J. Sound Vib. (to be published).

${ }^{13}$ R. A. Burdisso and C. R. Fuller, "Theory of feedforward controlled system eigenproperties", J. Sound Vib. 153(3), 437-451 (1992).

${ }^{14}$ R. A. Burdisso and C. R. Fuller, "Dynamic behavior of structuralacoustic systems in feedforward control of sound radiation," J. Acoust. Soc. Am. 92, 277-286 (1992).

${ }^{15}$ C. R. Fuller and R. A. Burdisso, "A wavenumber domain approach to the active control of structure-borne sound," J. Sound Vib. 148(2), 355-360 (1991). 